\title{
Search for dark sector via charmonia decay at BESIII
}

\section{X.D. Shi for the BESIII Collaboration*}

State Key Laboratory of Particle Detection and Electronics

University of Science and Technology of China

E-mail: 区iaodong.shiemail.ustc.edu.cn

Identifying the nature of dark matter is an unsolved problem in astronomy and particle physics. Although much evidence is observed in astronomical experiments, there is no evidence from collider experiments. Many theories beyond the Standard Model predict light dark matter, dark photon and dark gauge boson, which may be light enough for BESIII to observe. BESIII has recently searched for invisible decays of light mesons and dark photons as well as dark gauge bosons using the 1.3 billion $\mathrm{J} / \psi$ data sample. This poster summaries the recent results from BESIII collaboration on these dark sector topics.

XXIX International Symposium on Lepton Photon Interactions at High Energies - LeptonPhoton2019 August 5-10, 2019

Toronto, Canada

\footnotetext{
*Speaker.
} 


\section{Introduction}

Understanding dark matter is a major task of astronomy and particle physics. There is lots of evidence of dark matter from astronomical experiments. [四] Nevertheless, there is no evidence from any collider experiments until now. [] Many new physics theories beyond the Standard Model (SM) introduce the dark sector and predict the interaction between dark sector and quarkonium. [B]

BESIII [ [ 4 ], an $e^{+} e^{-}$collider experiment running at $\tau$-charm energy region with peak luminosity at $10^{33} \mathrm{~cm}^{-2} \mathrm{~s}^{-1}$, has accumulated 10 billion $\mathrm{J} / \psi$ events ${ }^{1}$, which provide an excellent opportunity to search for the dark sector via charmonia decay.

\section{Search for invisible decays of $\omega / \phi$}

In SM the invisible decays of quarkonium mainly happen by annihilating into a pair of neutrinos via a virtual $Z^{0}$ boson with an extremely small branching fraction. For example, $\mathscr{B}(\omega / \phi \rightarrow v \bar{v})$ $=(2.79 \pm 0.05) \times 10^{-13} /(1.67 \pm 0.02) \times 10^{-11}$. [目] But these processes could be enhanced up to the $10^{-8}$ level by light dark matter(LDM) particles. [6] BaBar, BESII and BESIII have searched for $\Upsilon(1 S), J / \psi$ and $\eta\left(^{\prime}\right)$ quarkonium invisible decay before, with no signal observation.[四]

BESIII has searched for the invisible decays of $\omega / \phi$ for the first time using 1.3 billion $\mathrm{J} / \psi$ events via $\mathrm{J} / \psi \rightarrow V \eta$ process, where $\eta$ is reconstructed by $\pi^{+} \pi^{-} \pi^{0}(\gamma \gamma)$. [8] The mass recoiling against $\eta, M_{\text {recoil }}^{V}=\sqrt{\left(E_{\mathrm{CM}}-E_{\pi^{+} \pi^{-} \pi^{0}}\right)^{2}-\vec{p}_{\pi^{+} \pi^{-} \pi^{0}}^{2}}$, is calculated to search for a signal. No significant signal is observed (Figure $\mathrm{Wleft}$ ). Using the Bayesian approach, the upper limits of the ratios at the $90 \%$ C.L. are calculated to be $\frac{\mathscr{B}(\omega \rightarrow \text { invisible })}{\mathscr{B}\left(\omega \rightarrow \pi^{+} \pi^{-} \pi^{0}\right)}<8.0 \times 10^{-5}$ and $\frac{\mathscr{B}(\phi \rightarrow \text { invisible })}{\mathscr{B}\left(\phi \rightarrow K^{+} K^{-}\right)}<3.4 \times 10^{-4}$ (Figure $\square$ middle and right). According to the world average values of $\mathscr{B}\left(\omega \rightarrow \pi^{+} \pi^{-} \pi^{0}\right)$ and $\mathscr{B}\left(\phi \rightarrow K^{+} K^{-}\right)$[Q] , the upper limits of $\omega$ and $\phi$ invisible decays at the $90 \%$ C.L. are set to be $7.2 \times 10^{-5}$ and $1.7 \times 10^{-4}$, respectively.
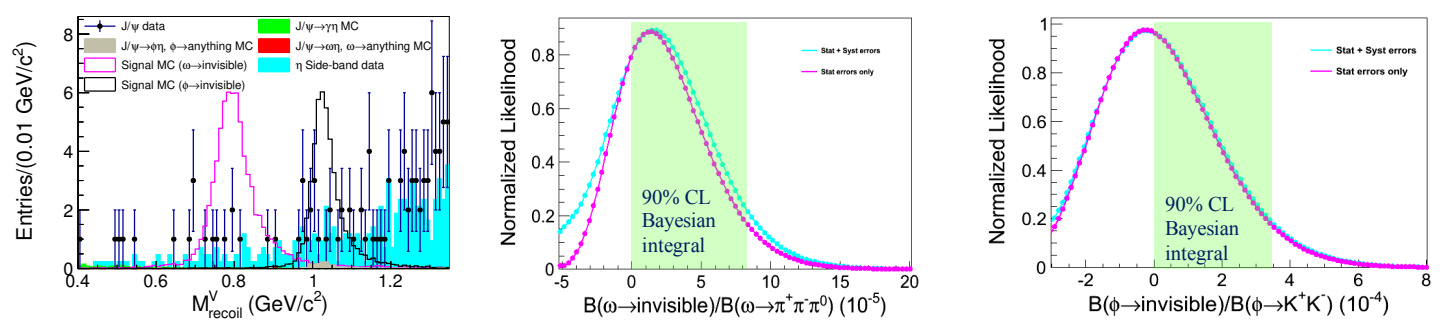

Figure 1: The $M_{\text {recoil }}^{V}$ distribution for data (black dots with error bars), signal MC samples (pink and black histograms for $\omega$ and $\phi$ decays, respectively) and various expected backgrounds shown by various colored histograms (left), and the normalized likelihood versus $\frac{\mathscr{B}(\omega \rightarrow \text { invisible })}{\mathscr{B}\left(\omega \rightarrow \pi^{+} \pi^{-} \pi^{0}\right)}$ (middle) and $\frac{\mathscr{B}(\phi \rightarrow \text { invisible })}{\mathscr{B}\left(\phi \rightarrow K^{+} K^{-}\right)}$(right) including the statistical uncertainty only (pink) and both systematic and statistical uncertainties (cyan).

\section{Search for dark photon}

The simplest scenario of beyond SM theory usually consist of an extra $U(1)$ gauge group, introducing a massive vector boson force carrier, the dark photon $\left(\gamma^{\prime}\right)$. The dark photon could couple

\footnotetext{
${ }^{1} 8.7$ billion $\mathrm{J} / \mathrm{psi}$ are collected in the recent two years.
} 
to the SM photon via the kinetic mixing strength, defined as $\varepsilon^{2}=\alpha^{\prime} / \alpha$, where $\alpha^{\prime}$ and $\alpha$ are the fine structure constants in the dark and SM sectors, respectively. [ए0] Thus the observed astrophysical anomalies [ $[$ ] could be explained, as well as the deviation in the muon anomalous magnetic moment up to the level of $(3-4) \sigma$ between theory and experiment [ㅁ]]. Many experiments have searched for the $\gamma^{\prime}$ and but only got an exclusion region on $\varepsilon$ so far. [12]

BESIII has recently searched for the $\gamma^{\prime}$ with electron position pair final states through $J / \psi \rightarrow$ $\gamma^{\prime} \eta\left({ }^{\prime}\right)$ using 1.3 billion of $J / \psi$ events, where $\eta\left(\eta^{\prime}\right)$ is reconstructed from $\eta \rightarrow \pi^{+} \pi^{-} \pi^{0}, \pi^{0} \rightarrow \gamma \gamma$ and $\eta \rightarrow \gamma \gamma\left(\eta^{\prime} \rightarrow \gamma \pi^{+} \pi^{-}\right.$and $\left.\eta^{\prime} \rightarrow \eta \pi^{+} \pi^{-}, \eta \rightarrow \gamma \gamma\right)$. [피] The search is performed in the $m_{e^{+}} e^{-}$distribution outside the $\omega$ and $\phi$ mass regions. No evidence of signal is found. As shown in Figure 凹, the $90 \%$ confidence level upper limits of branching fractions $\mathscr{B}\left(J / \psi \rightarrow \eta\left(^{\prime}\right) \gamma^{\prime}\right) \times \mathscr{B}\left(\gamma^{\prime} \rightarrow\right.$ $e^{+} e^{-}$) and $\varepsilon$ are set to be at the level of $10^{-8}$ and $10^{-3}$, respectively, depending on the mass of $\gamma^{\prime}$.
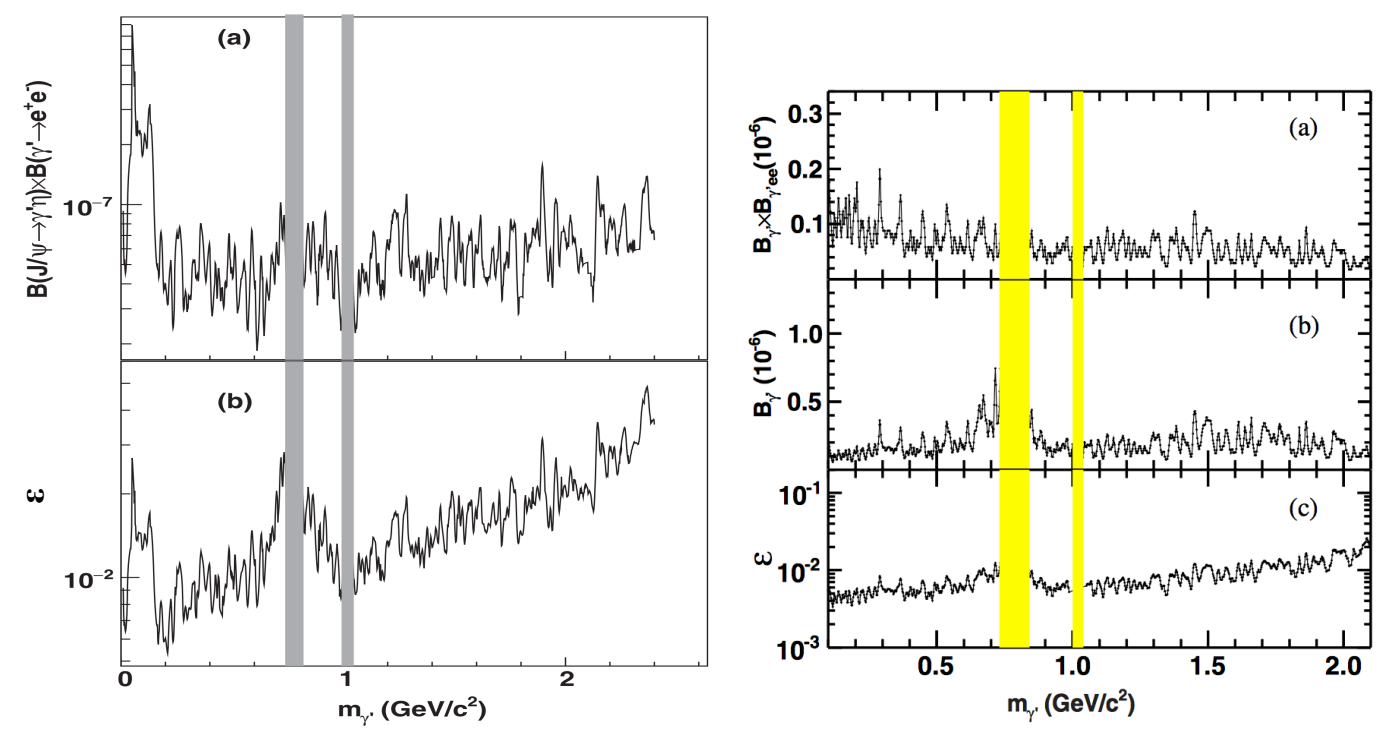

Figure 2: The 90\% C.L. upper limits on the branching fractions $\mathscr{B}\left(J / \psi \rightarrow \gamma^{\prime} \eta\left({ }^{\prime}\right)\right) \times \mathscr{B}\left(\gamma^{\prime} \rightarrow e^{+} e^{-}\right)$(top) and the kinetic mixing strength $\varepsilon$ (bottom). The left plots are for $J / \psi \rightarrow \gamma^{\prime} \eta$ decay and right plots for $J / \psi \rightarrow$ $\gamma^{\prime} \eta^{\prime}$ decay. The right hand side figure also shows the plot of $90 \%$ C.L. upper limit on $\mathscr{B}\left(J / \psi \rightarrow \gamma^{\prime} \eta^{\prime}\right)$.

\section{Search for dark gauge boson via $\mathbf{J} / \psi \rightarrow U^{\prime} \eta^{\prime}, U^{\prime} \rightarrow \gamma \pi^{0}$}

In the theory with an extra $U(1)$ gauge group metioned in the previous section, the new gauge boson named $U^{\prime}$ could predominantly couple to quarks in some mass region. [U4]] The Belle and KLOE Collaborations searched for this $U^{\prime}$ with $\pi^{+} \pi^{-}$daughter particles.[[5]]

BESIII recently searched the $U^{\prime}$ boson in the $\mathrm{J} / \psi \rightarrow U^{\prime} \eta^{\prime}, U^{\prime} \rightarrow \gamma \pi^{0}$ decay chain, where $\eta^{\prime}$ is reconstructed by $\pi^{+} \pi^{-} \eta(\gamma \gamma) .{ }^{2}$ The signal search is performed in the $m\left(\gamma \pi^{0}\right)$ distribution outside the $\omega$ mass region.No evidence of signal process is observed. Then the $90 \%$ C.L. upper limits on the product branching fraction $\mathscr{B}\left(J / \psi \rightarrow U^{\prime} \eta^{\prime}\right) \times \mathscr{B}\left(U^{\prime} \rightarrow \pi^{0} \gamma\right)$ is set at $10^{-7}$ level.

\footnotetext{
${ }^{2}$ This work is still preliminay.
} 


\section{Summary}

Recently BESIII has searched for invisible decays of light mesons and dark photons as well as dark gauge bosons using the 1.3 billion $\mathrm{J} / \psi$ data sample. No significant signal is found in these processes. Upper limits at $90 \%$ C.L. are set. BESIII is performing several other searches for dark sector using the large quarkonium data-sets. More results will be released in the near future.

\section{References}

[1] O. Adriani et al., Nature 458, 607-609 (2009); M. Aguilar et al. (AMS Collaboration), Phys. Rev. Lett. 110, 141102 (2013); J. Chang et al., Nature (London) 456, 362 (2008); M. Ackermann et al. (Fermi LAT Collaboration), Phys. Rev. Lett. 108, 011103 (2012); M. Aguilar et al. (AMS Collaboration), Phys. Rev. Lett. 110, 141102 (2013).

[2] G. Bertone, D. Hooper and J. Silk, Phys. Rept. 405, 279 (2005)

[3] N. Arkani-Hamed, D. P. Finkbeiner, T. R. Slatyer and N. Weiner, Phys. Rev. D 79, 015014 (2009); P. Fayet, Phys. Rev. D 75, 115017 (2007)

[4] M. Ablikim et al. [BESIII Collaboration], Nucl. Instrum. Meth. A 614, 345 (2010); C. Z. Yuan and S. L. Olsen, Nature Rev. Phys. 1 (2019) no.8, 480.

[5] D. N. Gao, Phys. Rev. D 98, 113006 (2018).

[6] B. McElrath, Phys. Rev. D 72, 103508 (2005); Light Higgses and Dark Matter at Bottom and Charm Factories, eConf C070805, 19 (2007).

[7] B. Aubert et al. (BaBar Collaboration), Phys. Rev. Lett. 103, 251801 (2009); M. Ablikim et al. (BES Collaboration), Phys. Rev. Lett. 100, 192001 (2008); M. Abilikim et al. (BESIII Collaboration), Phys. Rev. D 87, 012009 (2013).

[8] M. Abilikim et al. (BESIII Collaboration), Phys. Rev. D 98, 032001 (2018).

[9] M. Tanabashi et al. (Particle Data Group), Phys. Rev. D 98, 030001 (2018).

[10] N. Arkani-Hamed, D. P. Finkbeiner, T. R. Slatyer and N. Weiner, Phys. Rev. D 79, 015014 (2009).

[11] M. Pospelov, Phys. Rev. D 80095002 (2009).

[12] J. P. Lees et al. (BaBar Collaboration), Phys. Rev. Lett. 113, 201801 (2014); J. R. Batley et al. (NA48/2 Collaboration), Phys. Lett. B 746, 178 (2015); M. Ablikim et al. (BESIII Collaboration), Phys. Lett. B 774, 252 (2017).

[13] M. Ablikim et al. (BESIII Collaboration), Phys. Rev. D 99, 012006 (2019); Phys. Rev. D 99, 012013 (2019).

[14] A. E. Nelson and N. Tetradis, Phys. Lett. B 221 (1989) 80; S. Tulin, Phys. Rev. D 89 (2014) no.11, 114008

[15] E. Won et al. [Belle Collaboration], Phys. Rev. D 94 (2016) no.9, 092006; A. Anastasi et al. [KLOE-2 Collaboration], Phys. Lett. B 757 (2016) 356 